\title{
Conocimiento experto y autorregulación en adultos mayores jubilados profesionales y no profesionales
}

\author{
Expert Knowledge and Self-Regulation in Older Adults, Professional \\ and Mon-Professional Retirees \\ Conhecimento experto e auto-regulamentação em idosos aposentados \\ profissionais e não profissionais
}

\author{
Deisy Krzemien* \\ María M. Richard's* \\ *Consejo Nacional de Investigaciones Cientificas y Técnicas, Instituto de Psicología Básica, \\ Aplicada y Tecnología, Universidad Nacional de Mar del Plata, Buenos Aires, Argentina. \\ María Ayelén Biscarra** \\ **Consejo Nacional de Investigaciones Cientificas y Técnicas, Universidad Atlántida Argentina, \\ Universidad Nacional de Mar del Plata, Buenos Aires, Argentina.
}

Doi: http://dx.doi.org/10.12804/revistas.urosario.edu.co/apl/a.4793

\section{Resumen}

Si bien se han investigado variables psicosociales implicadas en la adaptación posjubilatoria, existe escaso interés en el factor del conocimiento experto. Este estudio propone comparar las metas de vida a partir del retiro jubilatorio en adultos mayores profesionales y no profesionales, y analizar la relación entre el conocimiento experto y las estrategias de autorregulación, con el fin de contrastar empíricamente el modelo del
Grupo de Berlín. Se utilizó un diseño correlacional transversal en una muestra de 258 jubilados distribuidos en dos grupos: profesionales y no profesionales. Se hallaron diferencias en la selección de metas entre ambos grupos y se evidenció que el conocimiento experto se relacionó con un mayor uso de estrategias de optimización y compensación. El grupo no profesional utilizó la estrategia compensación en mayor medida, y el grupo profesional presentó un desempeño significativamente mayor en conocimiento experto. Los datos

* Deisy Krzemien, María M. Richard's, Consejo Nacional de Investigaciones Científicas y Técnicas (Conicet), Instituto de Psicología Básica, Aplicada y Tecnología (Ipsibat), Facultad de Psicología, Universidad Nacional de Mar del Plata (UNMDP), Buenos Aires, Argentina.

** María Ayelén Biscarra, Consejo Nacional de Investigaciones Científicas y Técnicas, Universidad Atlántida Argentina, Universidad Nacional de Mar del Plata, Buenos Aires, Argentina.

La correspondencia relativa a este artículo debe ser enviada a Deisy Krzemien, Alsina 2556, $1^{\circ}$ G. CP 7600, Mar del Plata, Argentina. Correo electrónico: deisyk@conicet.gov.ar

Cómo citar este artículo: Krzemien, D., Richard's, M. M. \& Ayelén Biscarra, M. (2018). Conocimiento experto y autorregulación en adultos mayores jubilados profesionales y no profesionales. Avances en Psicología Latinoamericana, 36(2), 331 -344. DOI: http://dx.doi.org/10.12804/revistas.urosario.edu.co/apl/a.4793 
obtenidos sirven al diseño de programas de asistencia prejubilatoria.

Palabras clave: jubilación, autorregulación, conocimiento experto, metas de vida, adultos mayores.

\section{flbstract}

While various psychosocial variables, involved in post-retirement adaptation, were investigated, enough attention has not been paid to the role of expert knowledge. This research aims to compare life goals of professional and non-professional older adults since their retirement, and to analyze the relationship between the expert knowledge and the self-regulation strategies, in order to empirically evaluate the Berlin Group model. A cross-correlational design was used, with a sample of 258 participants, retirees, divided into two groups: professionals and non-professionals. Differences were found in the selection of life goals between both groups and it became clear that expert knowledge was associated with increased use of optimization and compensation strategies. The non-professional group used the compensation strategy more than the other group, and the professional group presented a significantly higher performance in expert knowledge than the non-professional group. The data obtained can be of use for the design of public policies and programs pre-retirement assistance.

Keywords: retirement, self-regulation, expert knowledge, life goals, older adults.

\section{Resumo}

Embora se investigaram variáveis psicossociais implicadas na adaptação pós-aposentadora, existe escasso interesse no fator do conhecimento experto. Este estudo propõe comparar as metas de vida a partir da aposentadoria em idosos profissionais e não profissionais, e analisar a relação entre o conhecimento experto e as estratégias de auto-regulamentação, com o fim de contrastar empiricamente o modelo do Grupo de Berlim. Se utilizou um desenho de correlação transversal, em uma amostra de 258 aposentados, distribuídos em dois grupos: profissionais e não profissionais. Encontraram-se diferenças na seção de metas entre ambos os grupos e se evidenciou que o conhecimento experto se relacionou com um maior uso de estratégias de optimização e compensação. O grupo não profissional apresentou um desempenho significativamente maior em conhecimento experto. Os dados obtidos servem ao desenho de programas de assistência pré-aposentadora. Palavras-chave: aposentadoria, auto-regulamentação, conhecimento experto, metas de vida, idosos.

\section{Introducción}

En el campo de la psicología del potencial humano, el estudio del proceso de autorregulación ha cobrado relevancia para la comprensión de la adaptación psicológica a las transiciones de vida que afrontan los adultos mayores (Aspinwall \& Staudinger, 2007; Berger, 2009). El retiro jubilatorio es una de las principales transiciones críticas del envejecimiento, para la cual no están especificadas tareas productivas normativas y a menudo amenaza la capacidad adaptativa en la vida cotidiana (Fernández-Ballesteros, 2011). Numerosas investigaciones en los últimos años se centraron en el impacto psicosocial de la jubilación (Aymerich, Planes \& Gras, 2010; Hermida \& Stefani, 2011; Madrid \& Garcés, 2000; Martínez, Flórez, Ancizu, Valdés \& Adeva, 2003). No obstante, los resultados obtenidos hasta el momento son diversos y hasta contradictorios, más aún cuando se tiene en cuenta la influencia de la heterogeneidad de las variables personales y psicosociales. Atchley y Robinson (1982) consideran que cuanto más cualificado y especializado es el trabajo y más identificada está la persona con su actividad laboral, más difícil es adaptarse a la nueva situación de retiro. La adaptación a las crisis vitales se supone consecuencia del uso de recursos de resiliencia y autorregulación (Serrano et al., 2001). Sin embargo, no está esclarecido cuáles son específicamente las estrategias de autorregulación utilizadas por los adultos 
mayores y cuál es su eficacia adaptativa (Carver \& Scheier, 2003).

Paul Baltes y Magret Baltes (1990) propusieron un modelo de adaptación (Action Auto-Regulation Theoretical Framework) basado en el principio de optimización selectiva con compensación. El Grupo de Berlín (Aspinwall \& Staudinger, 2007; Baltes \& Freund, 2003) sostiene que el buen envejecimiento implica esforzarse continuamente en minimizar las pérdidas —o sus efectos negativos-y maximizar los recursos. La idea principal es que el envejecimiento competente depende del interjuego entre tres estrategias de autorregulación: selección, optimización y compensación (SOC). Es decir, el proceso de adaptación incluye el esfuerzo de priorización de unas metas y restricción de otras, reforzar y potenciar los recursos, y compensar y regular las pérdidas asociadas al avance de la edad. Las estrategias compensatorias se desarrollan cuando se ve reducida la capacidad adaptativa, posibilitando el desarrollo potencial de recursos propios y del medio ambiente (Greve \& Staudinger, 2006) (tabla 1).

Varios investigadores relacionan el envejecimiento satisfactorio con la capacidad adaptativa, en particular con el uso de las estrategias SOC (Baltes \& Freund, 2007; Franklin \& Tate, 2009; Freund \& Baltes, 2002; Ziegelmann \& Lippke, 2007). Sin embargo, si bien el uso de recursos SOC se ha hallado asociado positivamente a indicadores de bienestar en adultos jóvenes (Wiese, Freund \& Baltes, 2000), los estudios empíricos en adultos mayores son aún exiguos (Lerner, Lerner, Almerigi \& Theokas, 2005). La investigación del modelo soc desde su formulación en 1990 ha sido más bien teórica; los estudios tempranos examinaron los comportamientos adaptativos de personas frente al padecimiento de disfuncionalidades y enfermedades crónicas (Gignac, Cott \& Badley, 2002; Marsiske, Lang, Baltes \& Baltes, 1995). Recientemente, el modelo soc fue aplicado al estudio de la adaptación a condiciones sociales y psicológicas críticas (Rush, Watts \& Stanbury, 2011). Por ejemplo, Lang, Rieckmann y Baltes (2002) hallaron que las personas mayores con muchos recursos utilizaban más frecuentemente las estrategias de selección y compensación, y que frente a situaciones adversas, se tiende a incrementar el uso de procesos de selección electiva y restrictiva. Abraham y Hansson (1995) han sugerido que el modelo soc puede ser útil para explicar cómo los trabajadores mayores pueden mantener competencias laborales luego de la jubilación.

Por otra parte, el mismo Grupo de Berlín se orienta al estudio del desarrollo ontogenético del conocimiento experto como sabiduría (Baltes \&

Tabla 1

Operacionalización del modelo Soc: optimización selectiva con compensación

\begin{tabular}{|c|c|c|}
\hline \multicolumn{3}{|c|}{ Estrategias adaptativas del envejecimiento exitoso } \\
\hline Selección & Optimización & Compensación \\
\hline $\begin{array}{l}\text { Selección electiva } \\
\text { - Especificación de metas. } \\
\text { - Sistema jerárquico de metas. } \\
\text { - Contextualización de metas. } \\
\text { - Compromiso en las metas. } \\
\text { Selección restringida } \\
\text { - Relegar y priorizar metas. } \\
\text { - Reconstrucción de la jerarquía de } \\
\text { metas. } \\
\text { - Sustitución de metas. } \\
\text { - Adaptación de estándares culturales. }\end{array}$ & $\begin{array}{l}\text { - Focalización en los dominios involu- } \\
\text { crados en las metas. } \\
\text { - Delimitar el tiempo oportuno. } \\
\text { - Persistencia. } \\
\text { - Adquirir y practicar habilidades. } \\
\text { - Generación y optimización de recur- } \\
\text { sos/medios. } \\
\text { - Esfuerzo. } \\
\text { - Distribución del tiempo. } \\
\text { - Aprendizaje y refuerzo. }\end{array}$ & $\begin{array}{l}\text { - Regulación de pérdidas. } \\
\text { - Uso de recursos externos y contextua- } \\
\text { les/ayuda instrumental. } \\
\text { - Intervención terapéutica. } \\
\text { - Activación o aprendizaje de nuevas } \\
\text { habilidades y recursos. } \\
\text { - Ponderación de la energía vital. } \\
\text { - Modelización. }\end{array}$ \\
\hline
\end{tabular}

Fuente: elaboración propia a partir de la propuesta del Grupo de Berlín (Freund \& Baltes, 2002; Lang et al., 2002) 
Staudinger, 2000). Se refiere a "un conocimiento experto y un juicio acerca de la pragmática fundamental de la vida" (Staudinger, 1999, p. 643). Se alude a la experiencia práctica que se evidencia en la resolución de problemáticas de la vida cotidiana. Los autores distinguen cinco criterios delimitadores del conocimiento experto: conocimiento práctico, conocimiento procedimental, contextualismo, relativismo y conciencia de la incertidumbre.

Baltes y Freund (2007) sostienen que el conocimiento experto y las estrategias SOC, cuando operan en conjunto, conducen a la adaptación y al desarrollo del potencial humano. Esta hipótesis se basa en la relación recíproca entre las estrategias SOC y el conocimiento experto. Aprender a seleccionar las metas, optimizarlas y compensar las pérdidas de tal modo que ayuden a obtener un desarrollo adaptativo debería facilitar un ajuste de metas más apropiadas para una persona o etapa vital y en un contexto histórico-social determinado. El modelo del soc describe un conjunto de estrategias de comportamiento por el cual las metas y los medios pueden ser perseguidos y logrados. En este sentido, la capacidad de adaptación se ve reforzada por una consideración conjunta del conocimiento experto como sabiduría y de las estrategias SOC (Baltes \& Freund, 2003).

Se han investigado diversas variables psicosociales implicadas en la adaptación posjubilatoria en la vejez, pero aún no se ha prestado suficiente atención a las variables cognitivas, como la experticia y la formación educativa especializada. Algunos factores que pueden influir en la habilidad de las personas para adaptarse a la transición jubilatoria son el conocimiento experto, la profesión y el nivel educativo. Como antecedentes en esta línea, se ha hallado relación entre el nivel educativo y el uso de estrategias de afrontamiento activas y cognitivas frente a la crisis de la jubilación (Krzemien, Urquijo \& Monchietti, 2004). Otros estudios hallaron que las personas, en la transición al retiro, con un mayor nivel profesional mantienen una mayor satisfacción con la jubilación (Fernández, Crego \& Alcover, 2008) y las personas jubiladas sin estudios o con estudios primarios experimentan niveles de depresión más altos que aquellos con estudios universitarios (Martínez et al., 2003). Los estudios del Grupo de Berlín (Smith, Staudinger \& Baltes, 1994; Staudinger, Smith \& Baltes, 1992) mostraron que personas con ciertas especializaciones profesionales alcanzan un alto nivel de conocimiento experto. En relación con la productividad, algunos estudios destacan la ausencia de una relación negativa con la edad. Se evidenció que, en gerentes de entidades bancarias de edad avanzada próximos a jubilarse, el conocimiento experto puede contribuir a compensar las pérdidas de funcionamiento cognitivo asociadas a la edad (Colonia-Willner, 1999).

A partir de los desarrollos presentados, este artículo propone los siguientes objetivos: (1) identificar y comparar las metas de vida a partir del retiro jubilatorio en adultos mayores profesionales y no profesionales; (2) establecer diferencias en el desempeño en el conocimiento experto y las estrategias de autorregulación SOC en ambos grupos; y (3) analizar la relación entre las variables de estudio según los grupos. En relación con estos objetivos, se proponen las siguientes hipótesis: (1) las metas de vida del grupo de los adultos mayores profesionales están vinculadas a su trayectoria profesional o centradas a su actividad previa, en tanto que las metas de los adultos mayores no profesionales se refieren a un amplio y variado conjunto de intereses; (2) las estrategias de autorregulación se hallan asociadas al conocimiento experto, por lo que los adultos mayores profesionales presentan niveles de experticia más elevados y un nivel alto de estrategias de autorregulación de selección, optimización y compensación ajustadas al logro de un comportamiento adaptativo; y (3) en el grupo de profesionales el conocimiento experto se relaciona con el uso de las estrategias de optimización y compensación, y en el grupo de no profesionales 
el conocimiento experto se relaciona con la estrategia de selección de metas vitales.

\section{Método}

\section{Diseño y participantes}

Se utilizó un diseño correlacional comparativo transversal de grupos naturales (Ato, López \& Benavente, 2013). La muestra estuvo compuesta por 258 adultos mayores jubilados, de ambos sexos, seleccionados mediante un muestreo no probabilístico incidental y distribuidos en dos grupos, según sean profesionales o no, en función de los datos obtenidos mediante un cuestionario de experticia. Dicho cuestionario fue elaborado ad hoc según el modelo conceptual de Ericsson (2008). Está conformado por tres preguntas abiertas, de carácter cualitativo, no estandarizado, y cuyo objetivo es identificar y discriminar a los adultos mayores profesionales y no profesionales, según los criterios de experticia establecidos por Ericsson: actividad laboral-ocupacional, formación académica -profesional, área de especialización, años de formación profesional, años de experiencia o práctica y principal producción científica/técnica/artística.

El grupo 1 está integrado por 125 profesionalesexpertos $(48,4 \%), 79$ mujeres y 46 varones, todos ellos expertos con educación formal desde el nivel básico al superior (primario, secundario, terciario o universitario) pero con ocupación laboral previa altamente calificada, desempeñada tanto de forma independiente o en relación de dependencia, y experiencia en un área de trabajo específica desarrollada a lo largo de su vida laboral activa. La selección de los expertos se basó en los criterios definidos por Ericsson (2008). El grupo 2 está compuesto por 133 adultos mayores no profesionales (51,6\%), 90 mujeres y 43 varones. Estos participantes contaban con educación básica primaria completa o media, con o sin ocupación previa, por ejemplo, amas de casa. Se consideraron los siguientes criterios de inclusión para integrar la muestra total: participantes autoválidos, exentos de trastornos cognitivos, psicopatológicos o inmovilidad física, y recientemente jubilados o con un máximo de cinco años posteriores al retiro jubilatorio efectivo. Se excluyeron personas que residan en geriátricos, que se hallen en tratamiento psiquiátrico, psicológico o de rehabilitación cognitiva, o que consuman alguna medicación psiquiátrica. La muestra se extrajo de instituciones y centros de la ciudad de Mar del Plata, Argentina, incluyendo organismos gubernamentales y no gubernamentales, de gestión pública y privada, de diversa índole: cultural, educativa, social, de salud y recreativa. También se incluyeron participantes que no tenían ninguna pertenencia institucional y fueron contactados de manera particular.

\section{Instrumentos}

1. Cuestionario sociodemográfico, elaborado ad hoc para indagar edad, nivel educativo, sexo, estado civil, lugar de residencia, tipo de hogar (uni/multipersonal) y conformación de grupo conviviente utilizado en estudios previos ( $\mathrm{Kr}$ zemien \& Richard's, 2011).

2. Encuesta de metas de vida, elaborada ad hoc basada en estudios previos (Wiese, Freund \& Baltes, 2000). Se les solicitó a los participantes que identificaran y enumeraran tres metas principales en orden de jerarquía que ellos se plantearan a partir del retiro jubilatorio.

3. SOC Questionnaire (cuestionario selección, optimización y compensación) de Baltes, Baltes, Freund \& Lang (1999), traducido al español y aplicado en adultos mayores marplatenses (Krzemien, 2014). Se utilizó la modalidad de "dominio específico", versión abreviada. Consta de 12 pares de ítems opuestos distribuidos en cuatro categorías: selección electiva, selección restrictiva, optimización y compensación, cada una compuesta por tres pares de ítems. Los ítems presentan dos tipos de respuestas diferentes: uno describe el comportamiento 
que refleja las estrategias SOC y el otro describe estrategias alternativas (no SOC). Se le solicita al participante que elija la respuesta que más se acerca a su modo de proceder. Las estrategias se miden en una escala de valores de tres niveles: 1. Bajo, 2. Medio y 3. Alto. Se obtiene una puntuación general y de cada subescala. Presenta adecuadas propiedades psicométricas, reportándose un $\alpha$ de Cronbach de 0,78, 0,72, 0,68 y 0,67 para cada escala respectivamente; y los coeficientes test-retest del cuestionario fueron 0,74 y 0,82 a través de cuatro semanas (Freund \& Baltes, 2002).

4. Wisdom-Related Expert Knowledge Scale (WRKS Scale) (escala del conocimiento experto), del Grupo de Berlín (Staudinger, Smith \& Baltes, 1994), traducida al español y aplicada en adultos mayores marplatenses (Krzemien \& Richard's, 2011). Evalúa el conocimiento y juicio experto a partir de cinco criterios delimitadores: (1) conocimiento práctico, (2) conocimiento procedimental, (3) contextualismo, (4) relativismo, (5) conciencia de incertidumbre. En este estudio se aplicaron dos tareas recomendadas por los autores para la vejez (tarea A: "Revisión de la vida" y tarea B: "Planificación de la vida"), las cuales presentan situaciones hipotéticas dilemáticas de la vida cotidiana. Los participantes deben expresar una reflexión (thinking-aloud) y dar una resolución escrita considerando lo que debiera, conviniera o pudiera hacer una persona. Las respuestas son calificadas en una escala ordinal de 5 puntos ( 1 bajo a 5 alto) en cada uno de los cinco criterios y en general. Presenta adecuadas propiedades psicométricas, reportándose un $\alpha$ de Cronbach de 0,71 a 0,96 entre todos los criterios (Staudinger et al., 1992, 1994).

\section{Procedimiento y análisis de datos}

La administración de los instrumentos se realizó en una sesión de 60 minutos, en forma individual, mediante condiciones estandarizadas, sistemáticas y una guía de orientación brindada por los investigadores a fin de asegurar la comprensión de las consignas y evitar datos faltantes. Se aseguró el consentimiento informado y la confidencialidad de los datos personales de los participantes, cuya inclusión en el estudio fue voluntaria y anónima. Se aplicaron técnicas de análisis de contenido para analizar las metas de vida, y pruebas de estadística descriptiva para caracterizar las variables de estudio y de estadística inferencial en función del tipo de distribución de las variables. Para el análisis de contenido se siguieron los lineamientos de Huberman y Miles (2000), quienes proponen tres subprocesos vinculados entre sí para realizar el análisis: (a) la reducción de datos, orientada a su selección y condensación; (b) la presentación de datos, orientada a la reflexión del investigador a través de presentaciones resumidas del material; y (c) la elaboración y verificación de conclusiones, utilizando técnicas para extraer significados de los datos (comparación/contraste, señalamiento de patrones, saliencia, búsqueda de casos negativos).

\section{Resultados}

Se describen los aspectos sociodemográficos de la muestra de estudio y a continuación se exponen los resultados en función de los objetivos planteados en la introducción del presente trabajo.

\section{Datos sociodemográficos}

- Grupo 1, denominado Profesional: la media de edad es 67,79 $(D T=6,62)$, variando entre 60 y 87 años. En cuanto al nivel de educación, el 74,4\% completó estudios terciarios o universitarios, el 19,2 \% terminó estudios secundarios, y el 6,4\% completó estudios primarios, quienes habían alcanzado el nivel de conocimiento experto según los criterios de inclusión de la muestra basados en la selección de expertos de Ericsson (2008). En cuanto al estado civil, el $48 \%$ 
está casado, el $15,2 \%$ se halla divorciado, el $28,8 \%$ es viudo y $8 \%$ mantiene la soltería. La mayoría $(62,4 \%)$ convive con algún familiar o pareja y el $37,6 \%$ vive solo. Respecto al lugar de residencia, el $64,8 \%$ vive en barrios de la ciudad y el $35,2 \%$ en la zona céntrica.

- Grupo 2, denominado No profesional: la media de edad es 71,53 $(D T=7,84)$, oscilando entre 60 y 93 años. El 51,9\% de este grupo cursó solo estudios primarios y el $48,1 \%$ estudios secundarios. En cuanto al estado civil, el $44,4 \%$ es viudo, el $34,6 \%$ se encuentra casado, el $12 \%$ divorciado y el $9 \%$ restante es soltero. El 49,6\% vive solo y el 50,4\% convive con algún familiar o pareja. Respecto al lugar de residencia, el $75,2 \%$ vive en barrios de la ciudad y el $24,8 \%$ en la zona céntrica.

\section{Metas de vida}

Con el propósito de alcanzar el primer objetivo, inicialmente se procedió a un análisis cualitativo utilizando la técnica de análisis de contenido tradicional de los datos recogidos a través de la encuesta de metas de vida, que posibilitó extraer de cada respuesta las diferentes unidades de significado relevantes. Sintéticamente, este análisis incluyó: reducción, disposición de datos y obtención de las categorías (tabla 2). Luego, se aplicó un análisis estadístico de porcentajes de utilización de las metas seleccionadas por cada grupo según el grado de importancia asignado a estas por los sujetos al tiempo presente de participación de esta investigación. Los resultados mostraron que, en el caso del grupo profesional, como meta principal el $19,2 \%$ eligió viajes y turismo, el 18,4\% prefirió actividades productivas y continuidad laboral, y el 15,2\% seleccionó cuidado personal y salud. El grupo no profesional mencionó como prioridad de las metas viajes y turismo $(24,1 \%)$, amistad, familia y amor (22,5\%), cuidado personal y salud (18\%), mientras que la meta de actividades productivas y continuidad laboral resultó ser una de las de menor importancia en este grupo $(6,8 \%)$ (tabla 3$)$.

Tabla 2

Detalle de metas de vida en adultos mayores jubilados

\begin{tabular}{cl}
\hline Metas de vida & \multicolumn{1}{c}{ Descripción } \\
\hline & $\begin{array}{l}\text { Pasear. Retornar a visitar sitios de } \\
\text { origen familiar. Conocer nuevos } \\
\text { Viajes y turismo } \\
\text { lugares anhelados en la juventud. } \\
\text { Explorar otras culturas y conocer } \\
\text { nuevas personas. }\end{array}$ \\
\hline
\end{tabular}

Disfrutar de la relación con los hijos y nietos. Compartir juegos, salidas y tiempo libre con familiares. Colaborar con la crianza y el cuidado de los nietos, aportando tiempo y reamor cursos. Afianzar relaciones vinculares y establecer nuevos vínculos de amistad. Integrarse a grupos sociales. Participar en reuniones sociales y familiares. Conformar pareja. Acompañar al cónyuge enfermo.

Control médico. Dieta alimentaria

Cuidado personal/ salud adecuada. Emprender actividades preventivas y saludables (caminata, actividad física, asistencia a talleres de memoria). Rehabilitación.

Realizar actividades autosatisfactorias y gratificantes. Disfrutar de tiempo libre. Ocuparse de actividecoración dades decorativas y arreglo de la vivienda. Descansar. Vivir sin responsabilidad de cumplir horarios.

Continuar trabajando. Conservar las actividades laborales u ocupacionales. Actualizarse en las incumActividades bencias y avances en la profesión. productivas/ Continuar generando productos o continuidad laboral proyectos. Continuar instruyéndose mediante cursos y talleres. Formar a otros, trasmitir el saber y la experiencia.

Asistir a talleres de pintura, cine y Actividad artística, debate, entrenamiento de memoria, cultural, educativa lectura a escolares, computación, etc. 


\begin{tabular}{ll}
\hline \multicolumn{1}{c}{ Metas de vida } & \multicolumn{1}{c}{ Descripción } \\
\hline $\begin{array}{l}\text { Trascendencia, } \\
\text { legado y } \\
\text { solidaridad }\end{array}$ & $\begin{array}{l}\text { Trasmisión de saberes y experien- } \\
\text { cias de vida a otros. Dejar un legado } \\
\text { generacional a la descendencia. Ser } \\
\text { solidario con los demás. }\end{array}$ \\
\hline
\end{tabular}

Tabla 3

Porcentajes de la selección principal de metas en adultos mayores jubilados profesionales y no profesionales

\begin{tabular}{lcc}
\hline & Grupo 1 & Grupo 2 \\
\hline \multicolumn{1}{c}{ Metas de vida } & $\%$ & $\%$ \\
\hline Viajes y turismo & 19,2 & 24,1 \\
$\begin{array}{l}\text { Actividades productivas/continuidad } \\
\text { laboral }\end{array}$ & 18,4 & 6,8 \\
Cuidado personal/salud & 15,2 & 18 \\
Actividad artística, cultural, & 13,6 & 7,5 \\
educativa & 12,8 & 22,6 \\
Amistad, familia y amor & 8 & 10,5 \\
Mejora económica o bienes & 6,4 & 9,8 \\
Recreación, descanso y decoración & 6,4 & 0,8 \\
\hline Trascendencia, legado, solidaridad & & \\
\hline
\end{tabular}

Nota: grupo 1: profesional. Grupo 2: no profesional.

\section{Conocimiento experto y estrategias de autorregulación SOC}

Con respecto al segundo objetivo, primero se obtuvieron los estadísticos descriptivos de tendencia central y dispersión del conocimiento experto, y de las estrategias SOC para la muestra total y para cada grupo por separado. Luego, se exploró el tipo de distribución (K-S), y en función de ello se aplicó un análisis de diferencias de medias para distribuciones no paramétricas (prueba $U$ de Mann-Whitney) entre los grupos profesionales y no profesionales, en el caso de las dimensiones del conocimiento experto. Respecto a las estrategias de autorregulación SOC, se utilizó la prueba $t$ de diferencia de medias debido a su distribución normal, hallándose diferencias estadísticamente significativas en todos los criterios, excepto para los tipos de selección electiva y restrictiva. Las estrategias de optimización y compensación son mayormente utilizadas por el grupo no profesional, presentando diferencias estadísticamente significativas con el grupo de profesionales (tabla 4).

Tabla 4

Descriptivos y diferencias de medias de la frecuencia de uso de las estrategias Soc y del desempeño en conocimiento experto, discriminados para la muestra total y para cada grupo

\begin{tabular}{|c|c|c|c|c|c|}
\hline & Ambos grupos & Grupo 1 & Grupo 2 & $t$ & K-S \\
\hline Estrategias SOC & $\mathrm{M}(\mathrm{DE})$ & $\mathrm{M}(\mathrm{DE})$ & $\mathrm{M}(\mathrm{DE})$ & & \\
\hline Selección electiva (SE) & $1,44(0,37)$ & $1,42(0,38)$ & $1,45(0,36)$ &,- 67 & ,94 \\
\hline Selección restrictiva (SR) & $1,55(0,33)$ & $1,54(0,32)$ & $1,57(0,34)$ &,- 80 & ,95 \\
\hline Optimización (O) & $1,32(0,35)$ & $1,28(0,31)$ & $1,37(0,38)$ & $-2,02 *$ & ,21 \\
\hline Compensación (C) & $1,41(0,33)$ & $1,36(0,33)$ & $1,47(0,32)$ & $-2,68 * *$ &, 09 \\
\hline Criterios conocimiento experto & & & & $\mathrm{U}$ & \\
\hline 1 & $2,91(0,88)$ & $3,10(0,89)$ & $2,74(0,84)$ & $6212,50 * *$ &, 00 \\
\hline 2 & $2,70(0,86)$ & $2,96(0,86)$ & $2,45(0,79)$ & $5418,50 * *$ &, 00 \\
\hline 3 & $2,62(0,92)$ & $2,87(0,99)$ & $2,38(0,79)$ & $5784,00 * *$ &, 00 \\
\hline 4 & $1,71(0,87)$ & $3,00(0,85)$ & $2,43(0,81)$ & $5364,00 * *$ &, 00 \\
\hline 5 & $2,65(0,90)$ & $2,89(0,94)$ & $2,42(0,79)$ & $5812,00 * *$ &, 00 \\
\hline
\end{tabular}

Notas: Grupo 1: profesional. Grupo 2: no profesional. $U$ : prueba U de Mann-Whitney; $t$ : prueba t para muestras independientes. Criterios de conocimiento experto: (1) conocimiento práctico, (2) conocimiento procedimental, (3) contextualismo, (4) relativismo, (5) conciencia de la incertidumbre.

$* \mathrm{p} \leq 0,05 ; * \mathrm{p} \leq 0,01$. 
Con respecto al conocimiento experto en general, se evidenció que el desempeño promedio de la muestra total osciló entre los valores que caracterizan un nivel medio-alto de conocimiento experto (valores superiores a 2,50 - de 2,65 a 2,91-, según la puntuación alcanzada en el instrumento), excepto en el caso del criterio relativismo que está por debajo de la puntuación media. Las puntuaciones promedio más altas se obtuvieron en conocimiento práctico y procedimental, luego en contextualismo y conciencia de la incertidumbre. Respecto al grupo profesional, el desempeño en todos los criterios de conocimiento experto fue superior al nivel medio, mientras que el grupo no profesional solamente logró un desempeño superior al nivel medio en el criterio conocimiento práctico.

Respecto al tercer objetivo, referido a la contrastación de la hipótesis correlacional entre el conocimiento experto y las estrategias SOC según cada grupo, se calculó el coeficiente de correlación $r$ de Pearson (en el caso de las dimensiones del conocimiento experto se estandarizaron los valores de aquellas variables con una distribución no normal). Los resultados mostraron que en el grupo de profesionales se presentaron algunas correlaciones positivas: la optimización se relacionó con los criterios conocimiento práctico $(\mathrm{r}=0,17 ; \mathrm{p} \leq, 05)$ y contextualismo $(\mathrm{r}=0,18 ; \mathrm{p} \leq, 05)$, y la compensación correlacionó con los criterios relativismo $(\mathrm{r}=0,17 ; \mathrm{p} \leq, 05)$ y contextualismo $(\mathrm{r}=0,19$; $\mathrm{p} \leq, 05)$ (tabla 5). Al contrario, en el grupo de no profesionales, se observaron correlaciones positivas significativas entre selección electiva y todos los criterios de conocimiento experto (tabla 6).

\section{Discusión}

En general, este artículo se inscribe en el debate teórico actual en el campo de la psicología del potencial humano acerca de la relación entre variables cognitivas y adaptativas. A pesar de que el modelo del Grupo de Berlín cuenta con solidez conceptual y suficiente consenso teórico en la literatura científica, la investigación empírica aún es exigua. Así, esta investigación intenta aportar evidencia empírica a favor de este modelo sobre la relación entre dos de los constructos clave del modelo, el conocimiento experto y las estrategias de autorregulación SOC (Baltes \& Freund, 2007), en adultos mayores, diferenciando entre profesionales y no profesionales. Los datos obtenidos revelan la consistencia parcial de dicho modelo, ya que se halló apoyo empírico respecto a las variables

Tabla 5

Correlaciones entre conocimiento experto y estrategias SOC en el grupo profesional

\begin{tabular}{|c|c|c|c|c|c|c|c|c|c|}
\hline & 1 & 2 & 3 & 4 & 5 & 6 & 7 & 8 & 9 \\
\hline 1. Conocimiento práctico & &, $77 * *$ &, $73 * *$ &, $57 * *$ &, $68 * *$ &,- 05 &,$- 26^{* *}$ &, $17^{*}$ &, 07 \\
\hline 2. Conocimiento procedimental & & &, $64 * *$ &, $54 * *$ &, $52 * *$ &,- 06 &,$- 19 *$ &, 10 &, 13 \\
\hline 3. Contextualismo & & & &, $59 * *$ &, $66^{* *}$ &, 00 &,$- 23 * *$ &, $18^{*}$ &, $19^{*}$ \\
\hline 4. Relativismo & & & & &, $69 * *$ & 01 &,- 13 &, $24 * *$ &, $17^{*}$ \\
\hline 5. Conciencia de incertidumbre & & & & & &, 05 &,- 17 &, 12 &, 12 \\
\hline 6. Selección electiva & & & & & & &, $31 * *$ &, $28 * *$ & ,09 \\
\hline 7. Selección restrictiva & & & & & & & &, 05 & ,09 \\
\hline 8. Optimización & & & & & & & & &, $49 * *$ \\
\hline 9. Compensación & & & & & & & & & \\
\hline
\end{tabular}

Nota: $* \mathrm{p} \leq 0,05 ; * * \mathrm{p} \leq 0,01$. 
Tabla 6

Correlaciones entre conocimiento experto y estrategias SOC en el grupo no profesional

\begin{tabular}{|c|c|c|c|c|c|c|c|c|c|}
\hline & 1 & 2 & 3 & 4 & 5 & 6 & 7 & 8 & 9 \\
\hline 1. Conocimiento práctico & &, $65 * *$ &, $60 * *$ &, $26 * *$ &, $51 * *$ &, $24 * *$ & ,08 &, 16 & , 16 \\
\hline 2. Conocimiento procedimental & & &, $55 * *$ &, $38 * *$ &, $39 * *$ &, $27 * *$ & ,03 &, 10 & ,11 \\
\hline 3. Contextualismo & & & &, $41 * *$ &, $56^{* *}$ &, $26 * *$ &, 06 &, 16 & ,08 \\
\hline 4. Relativismo & & & & &, $43 * *$ &, $17^{*}$ &, 10 &, 08 & ,08 \\
\hline 5. Conciencia de incertidumbre & & & & & &, 16 &, 04 &, 15 &, 08 \\
\hline 6. Selección electiva & & & & & & &, $21 *$ &, 10 &, 08 \\
\hline 7. Selección restrictiva & & & & & & & &, $23 * *$ & , 14 \\
\hline 8. Optimización & & & & & & & & &, $54 * *$ \\
\hline 9. Compensación & & & & & & & & & \\
\hline
\end{tabular}

Nota: $* \mathrm{p} \leq 0,05 ; * * \mathrm{p} \leq 0,01$.

de estudio consideradas de forma independiente; no obstante, no se ha encontrado evidencia empírica al considerar las interacciones entre dichas variables. De todas maneras, los autores advierten que conocimiento y comportamiento están lejos de correlacionar; saber algo no necesariamente implicará que actuemos en consecuencia, con la mejor de las estrategias (Baltes \& Freund, 2007). Una cuestión fundamental, entonces, es determinar cómo se puede adquirir una sabiduría relacionada con el conocimiento experto que se pueda trasladar a acciones y comportamientos adaptativos para con uno mismo y con los otros, y favorecer el desarrollo potencial en la vejez (Aspinwall \& Staudinger, 2007).

En primer lugar, este estudio se orientó a esclarecer la selección de metas de vida frente a la situación del retiro jubilatorio, en adultos mayores con y sin formación profesional. Las metas viajes y turismo y cuidado personal y salud fueron elegidas por ambos grupos; no obstante, la meta de actividad productiva y continuidad laboral fue seleccionada preponderantemente por los profesionales, mientras que la meta de amistad, familia y amor fue elegida principalmente por los no profesionales. Esto coincide con la postura de Nurmi (1992) para quien las metas de vida se refieren a propósitos, intereses y eventos futuros anticipados, que se relacionan con las tareas y las preocupaciones propias a lo largo del curso vital. Es esperable que aquellos adultos mayores con una trayectoria profesional prioricen metas relativas a su ocupación, mientras que las personas no profesionales tienden a valorar propósitos de vida relativos a la preservación de las relaciones interpersonales y familiares en la etapa posjubilatoria. Coincidentemente con otros estudios (Kim \& Feldman, 2000; Pastor et al., 2003), la forma en que las personas se adaptan a la jubilación depende en gran medida de las estrategias de autorregulación de las que disponga, además de otras variables psicosociales y macrosociales condicionantes. En este sentido, siguiendo el modelo SOC, es importante el reajuste de metas, seleccionar qué metas priorizar, a medida que varían o se limitan los recursos durante el curso vital (Wrosch, Scheier, Miller, Schulz \& Carver, 2003).

En segundo lugar, con respecto a las diferencias observadas entre los grupos, en cuanto al uso de las estrategias SOC, las personas no profesionales tienden a usar preferentemente la compensación y la optimización. Debido a que poseen menor formación profesional como recurso adaptativo, se valen de otros medios de manera compensatoria 
frente al retiro jubilatorio. En cuanto a las variables cognitivas, los datos mostraron que los profesionales alcanzan un desempeño significativamente mayor en todas las dimensiones del conocimiento experto en relación con los adultos mayores no profesionales. En coincidencia con los estudios previos del Grupo de Berlín (Smith et al., 1994; Staudinger et al., 1992), en esta investigación se evidenció el importante papel de la formación profesional en el desempeño en conocimiento experto.

En tercer lugar, al analizar las correlaciones entre las estrategias adaptativas y cognitivas dentro de cada grupo, se observó que, por un lado, en el grupo de los adultos mayores profesionales, el conocimiento experto se relaciona con el uso de las estrategias de optimización y compensación, como es esperable según el modelo conceptual del Grupo de Berlín. Es decir, los jubilados profesionales presentan una mayor capacidad para optimizar los recursos provenientes del entrenamiento y los aprendizajes previos, y para compensar las limitaciones o pérdidas ligadas al abandono de la actividad laboral. Por otro lado, en el caso de los no profesionales, el conocimiento experto se relaciona con la selección electiva, es decir, cuanto mayor conocimiento posean las personas sin o con escasa formación profesional, mayor será la posibilidad de seleccionar entre una variedad amplia de metas e intereses heterogéneos.

En definitiva, a partir de los datos obtenidos en esta muestra de adultos mayores, se observa que las variables cognitivas (criterios de conocimiento experto) y las adaptativas (estrategias de autorregulación SOC) se hallan relacionadas en el caso de los profesionales. Específicamente, las personas con formación profesional que poseen conocimiento experto tienden a hacer uso de las estrategias adaptativas de optimización y compensación ante una situación de crisis, como el retiro jubilatorio. En particular, en el caso de personas profesionales, los datos evidenciaron que los criterios de conocimiento práctico, contextualismo y relativismo se relacionan en forma positiva con la optimización y la compensación. Es decir, los adultos mayores que cuentan con una rica reserva de saberes y experiencias de vida, que consideran los condicionamientos contextuales y circunstanciales, y que muestran tolerancia de las diferencias interindividuales y reconocimiento de valores tienden a optimizar sus recursos de autorregulación y a encontrar formas de compensar las limitaciones y pérdidas en la etapa posjubilatoria. En adelante, se plantea la necesidad de profundizar la investigación en esta línea, en diversas muestras de adultos mayores, a fin de contrastar la hipótesis propuesta por el Grupo de Berlín.

Los datos obtenidos sirven al diseño de programas de asistencia prejubilatoria que contemplen las diferencias individuales en cuanto a la formación y la especialización profesional, y que potencien los recursos adaptativos del adulto mayor, con la consiguiente reducción de los costos en seguridad social y salud pública. Esto cobra especial interés considerando que en la actualidad numerosos cargos directivos/gerenciales en diferentes ámbitos son ocupados por adultos mayores expertos o profesionales, próximos a retirarse de su actividad laboral. Al respecto, Alcover y Crego (2008) insisten en la necesidad de superar las categorizaciones tradicionales en la investigación sobre el retiro e incorporar otros factores específicos que consideren la pluralidad de la población trabajadora, incluyendo la planificación de la continuidad laboral o el empleo puente (bridge employment).

Actualmente, las organizaciones gubernamentales de seguridad social de algunos países están contemplado una serie de cambios en sus políticas de recursos humanos que puedan aliviar considerablemente las presiones económicas de una población que envejece y aumentar los niveles de productividad, como introducir, por vía reglamentaria, planes de prejubilación, fomentar trabajo de calidad a edades más avanzadas y retrasar la edad para el retiro, considerando en particular el caso de trabajadores mayores competentes o expertos (Butrica, Smith \& Steuerle, 2006). Por tanto, sería 
conveniente reorientar las políticas públicas, y ofrecer programas institucionales y comunitarios de planificación e intervención prejubilatoria tendientes a fomentar los recursos de optimización selectiva con compensación en adultos mayores, en beneficio del desarrollo de sociedades inclusivas de individuos de todas las edades.

\section{Referencias}

Abraham, J. D. \& Hansson, R. O. (1995). Successful aging at work: an applied study of selection, optimization, and compensation through impression management. Journal of Gerontology, 50(2), 94-103.

Alcover, C. M. \& Crego, A. (2008). Modalidades de retiro laboral en Europa: bienestar psicológico y factores psicosociales asociados. Revista de Psicología del Trabajo y de las Organizaciones, 24(3), 1576-1962.

Aspinwall, L. G. \& Staudinger, U. M. (eds.) (2007). Psicología del potencial humano. Cuestiones fundamentales y normas para una psicología positiva. Barcelona: Gedisa.

Atchley, R. C. \& Robinson, J. (1982). Attitudes toward retirement and distance from the event. Research on Aging, 4(3), 299-313.

Ato, M., López, J. J. \& Benavente, A. (2013). Un sistema de clasificación de los diseños de investigación en psicología. Anales de Psicologia, 29(3), 1038-1059. DOI: http://dx.doi. org/10.6018/analesps.29.3.178511

Aymerich, M., Planes, P. M. \& Gras, M. E. (2010). La adaptación a la jubilación $y$ sus fases: afectación de los niveles de satisfacción $y$ duración del proceso adaptativo. Anales de Psicología, 26(1), 80-88.

Baltes, P. B. \& Baltes, M. (1990). Psychological perspectives on successful aging: the model of selective optimitzation with compensation. En P. Baltes \& M. Baltes (eds.), Successful aging: perspectives from behavioral sciences (pp. 1-34). Cambridge: Cambridge University Press.

Baltes, P. B. \& Freund, A. M. (2003). The intermarriage of wisdom and selective optimization with compensation (SOC): two meta-heuristics guiding the conduct of life. En C. L. M. Keyes \& J. Haidt (eds.), Flourishing: positive psychology and the life well-lived (pp. 249-273). Washington, DC: American Psychological Association.

Baltes, P. B. \& Freund, A. M. (2007). El potencial humano como orquestación de la sabiduría y la optimización selectiva con compensaciones. En L. G. Aspinwall \& U. M. Staudinger (eds.), Psicología del potencial humano. Cuestiones fundamentales y normas para una psicología positiva (pp. 45-62). Barcelona: Gedisa.

Baltes, P. B. \& Staudinger, U. M. (2000). Wisdom: a meta-heuristic (pragmatic) to orchestrate mind and virtue toward excellence. American Psychology, 55(1), 122-136.

Baltes, P. B., Baltes, M. M., Freund, A. M. \& Lang, F. R. (1999). The measurement of selection, optimization, and compensation (SOC) by self report: technical report 1999. Berlín: Max-Planck-Institut für Bildungsforschung.

Berger, S. (2009). Psicología del desarrollo. Adultez $y$ vejez. Madrid: Panamericana.

Butrica, B. A., Smith, K. E. \& Steuerle C. E. (2006). Working for a good retirement: the retirement project. Washington, DC: The Urban Institute.

Carver, C. S. \& Scheier, M. S. (2003). Self-regulatory perspectives on personality. En T. Millon \& M. J. Lerner (eds.), Handbook of psychology: personality and social psychology Vol. 5 (pp. 185-208). Nueva Jersey: Wiley.

Colonia-Willner, R. (1999). Investing in practical intelligence: ageing and cognitive efficiency among executives. International Journal of Behavioral Development, 23(3), 591-614. DoI: 10.1080/016502599383711

Ericsson, K. A. (2008). Deliberate practice and acquisition of expert performance: a general overview. 
Academic Emergency Medicine, 15(11), 988994.

Fernández, J., Crego, A. \& Alcover, C. (2008). Relaciones entre factores sociodemográficos, motivación hacia el retiro temprano y satisfacción en la vida postlaboral. Revista de Psicología del Trabajo y de las Organizaciones, 24(3), 135-146.

Fernández-Ballesteros, R. (2011). Limitaciones y posibilidades de la edad. En Imserso. Libro blanco del envejecimiento activo (pp. 113-148). Madrid: Imserso.

Franklin, N. C. \& Tate, C. A. (2009). Lifestyle and successful aging: an overview. American Journal of Lifestyle Medicine, 3(1), 6-11. DOI: 10.1177/1559827608326125

Freund, A. \& Baltes, P. B. (2002). Life-management strategies of selection, optimization and compensation: measurement by self-report and construct validity. Journal of Personality and Social Psychology, 82, 642-662. DOI: 10.1037/0022-3514.82.4.642

Gignac, M., Cott, C, \& Badley, E. (2002). Adaptation to disability: applying selective optimization with compensation to the behaviors of older adults with osteoarthritis. Psychology and Aging, 17(3), 520-524.

Greve, W. \& Staudinger, U. M. (2006). Resilience in later adulthood and old age: ressources and potentials for successful aging. En D. Cicchetti \& D. Cohen (eds.), Developmental psychopatho$\log y\left(2^{\mathrm{a}}\right.$ ed.) (pp. 796-840). Nueva York: Wiley.

Hermida, P. \& Stefani, D. (2011). La jubilación como un factor de estrés psicosocial. Perspectivas en Psicología, 8, 101-107.

Huberman, A. \& Miles, M. (2000). Métodos para el manejo y el análisis de datos. En C. Denman y J. Haro (comp.), Por los rincones. Antología de métodos cualitativos en la investigación social (pp. 253-300). Hermosillo: El Colegio de Sonora.

Kim, S. \& Feldman, D. C. (2000). Working in retirement: the antecedents and consequences of bridge employment and its consequences for quality of life in retirement. Academy of Management Journal, 43, 1195-1210.

Krzemien, D. (2014). Sabiduría, pensamiento postformal y auto-regulación en adultos mayores. Estudos Interdisciplinares sobre o Envelhecimento, 19(2), 549-567.

Krzemien, D. \& Richard's, M. (2011). Sabiduría en adultos mayores. Un estudio comparativo de dos modelos. En M. C. Richaud \& V. Lemos (comps.), Psicología y otras ciencias del comportamiento Vol. I (pp. 173-196). Buenos Aires: Ediciones CIIPME-Conicet.

Krzemien, D., Urquijo, S. \& Monchietti, A. (2004). Aprendizaje social y estrategias de afrontamiento a los sucesos críticos del envejecimiento femenino. Psicothema, 16(3), 350-356.

Lang, F., Rieckmann, N. \& Baltes, M. (2002). Adapting to aging losses: do resources facilitate strategies of selection, compensation, and optimization in every functioning? Journal of Gerontology: Psychological Sciences, 57B, 501-509.

Lerner, R. M., Lerner, J. V., Almerigi, J. \& Theokas, C. (2005). Positive youth development: a view of the issues. Journal of Early Adolescence, 25(1), 10-16.

Madrid, A. J. \& Garcés, E. J. (2000). La preparación para la jubilación: revisión de los factores psicológicos y sociales que inciden en un mejor ajuste emocional al final del desempeño laboral. Anales de Psicología, 16(1), 87-99.

Marsiske, M., Lang, F., Baltes, M. \& Baltes, P. B. (1995). Selective optimization with compensation: life-span perspectives on successful human development. En R. A. Dixon \& L. Bäckman (eds.), Compensation for psychological defects and declines: managing losses and promoting gains (pp. 35-79). Mahwah: Lawrence Erlbaum.

Martínez, P. C., Flórez, J. A., Ancizu, I., Valdés, C. A. \& Adeva, J. (2003). Repercusiones psicológicas y sociales de la prejubilación. Psicothema, 15(1), 49-53.

Nurmi, J. E. (1992). Age differences in adult life goals, concerns, and their temporal extension: 
A life course approach to future-oriented motivation. International Journal of Behavioral Development, 15, 487-508.

Pastor, E., Villar, F., Boada, J., López, S., Varea, M. D. \& Zaplana, T. (2003). Significados asociados a la jubilación e influencia con la actividad de ocio y la ética del trabajo. Revista Multidisciplinar de Gerontología, 13(1), 15-22.

Rush, K. L., Watts, W. E. \& Stanbury, J. (2011). Mobility adaptations of older adults: a secondary analysis. Clinical Nursing Research, 20(1), 81100. DOI: 10.1177/1054773810379401

Serrano-Parra, M. D., Garrido-Abejar, M., Notario-Pacheco, B., Bartolomé-Gutiérrez, R., Solera-Martínez, M. \& Martínez-Vizcaíno, V. (2012). Validez de la escala de Resiliencia de Connor-Davidson (CD-RISC) en una población de mayores entre 60 y 75 años. International Journal of Psychological Research, 5(2) 49-57.

Smith, J., Staudinger, U. M. \& Baltes, P. B. (1994). Occupational settings facilitative of wisdom-related knowledge: the sample case of clinical psychologists. Journal of Consulting and Clinical Psychology, 62, 989-1000.

Staudinger, U. M. (1999). Older and wiser? Integrating results on the relationship between age and wisdom-related performance. International Journal of Behavioral Development, 23(3), 641664. DOI: 10.1080/016502599383739
Staudinger, U. M., Smith, J. \& Baltes, P. B. (1992). Wisdom-related knowledge in a life review task: age differences and the role of professional specialization. Psychology and Aging, 7(2), 271-281. DOI: 10.1177/0146167203254506

Staudinger, U. M., Smith, J. \& Baltes, P. B. (1994). Manual for the assessment of wisdom related knowledge and judgment. Berlín: Max Planck Institute for Human Development.

Wiese, B. S., Freund, A. M. \& Baltes, P. B. (2000). Selection, optimization and compensation: an action-related approach to work and partnership. Journal of Vocational Behavior, 57, 273-300. DOI: 10.1006/jvbe.2000.1752

Wrosch, C., Scheier M. F., Miller G. E., Schulz R. \& Carver, C. S. (2003). Adaptive self-regulation of unattainable goals: goal disengagement, goal reengagement, and subjective well-being. Personality and Social Psychology Bulletin, 29(12), 1494-1508.

Ziegelmann, J. P. \& Lippke, S. (2007). Use of selection, optimization and compensation strategies in health self-regulation: interplay with resources and successful development. Journal of Aging and Health, 19(3), 500-518. DOI: $10.1177 / 0898264307300197$

\section{Recibido: abril 17, 2016 Aprobado: enero 15, 2018}

